\title{
Fünf Jahre AAC-Netzwerk: Ein digitales System zur Amtshilfe bei der Lebens- und Futtermittelkontrolle im Europäischen Wirtschaftsraum
}

\section{Five years of AAC network: a digital system for administrative assistance in food and feed control on the European Market}

\author{
Christine Schweigel ${ }^{1}$ (D) Nikolčo Jagurinoski ${ }^{1} \cdot$ Annett Mellenthin $^{1}$
}

Received: 26 August 2021 / Accepted: 7 October 2021

(c) The Author(s) 2021, corrected publication 2021

\section{Zusammenfassung}

Das Netzwerk für Amtshilfe und Zusammenarbeit (Administrative Assistance and Cooperation, kurz AAC) ist gemäß Titel IV der Verordnung (EU) 2017/625 von den Kontrollbehörden der Mitgliedstaaten zu verwenden, um Informationen zu Verstößen in der Lebensmittelproduktionskette auszutauschen. Nach einer erfolgreichen Einsatzphase im Bereich Lebensmittelbetrug wurde 2016 eine Onlineplattform zum Informationsaustausch in der Allgemeinen Amtshilfe initiiert, um einfache Verstöße gegen das Lebensmittel- und Futtermittelrecht schneller adressieren zu können. Seitdem arbeitet das Netzwerk auf Grundlage eines effizienten, digitalen Informationsmanagementsystems. Der vorliegende Artikel beleuchtet Hintergründe und Entwicklung des behördeninternen Systems.

Schlüsselwörter Amtshilfe und Zusammenarbeit (AAC) · IMSOC-Verordnung · Lebensmittelbetrug · Grenzüberschreitende Verstöße $\cdot$ Lebens- und Futtermittelüberwachung

\begin{abstract}
In accordance with Title IV of Regulation (EU) 2017/625, the Administrative Assistance and Cooperation (AAC) network must be used by control authorities of the Member States, in order to exchange information on non-compliances in the food production chain. After a successful implementation phase in the area of food fraud, an online platform for the exchange of administrative information among the Member States was initiated in 2016. The goal was to address simple food and feed law non-compliances more quickly. Since then, the network has been operating as an efficient, digital information management system. In this article, the background and the development of the intra-authority system are presented.
\end{abstract}

Keywords Administrative Assistance and Cooperation (AAC) $\cdot$ IMSOC regulation $\cdot$ Food fraud $\cdot$ Cross-border infringements $\cdot$ Food and feed control $\cdot$ European market

\section{Hintergrund}

Im Jahr 2013 wurde als Reaktion auf den Pferdefleischskandal das europäische Netzwerk für Lebensmittelbetrug (,Food Fraud Network“, kurz FFN) gegründet. Der Vorfall offenbarte,

Christine Schweigel

christine.schweigel@bvl.bund.de

1 Federal Office of Consumer Protection and Food Safety, Berlin, Germany dass erheblicher Verbesserungsbedarf bei der europäischen Zusammenarbeit in Bezug auf vorsätzliche Verstöße mit grenzüberschreitenden Auswirkungen bestand. Bald zeigte sich die Notwendigkeit, den benannten nationalen Verbindungsstellen für Lebensmittelbetrug ein elektronisches Tool an die Hand zu geben, um den bis dahin üblichen Informationsaustausch per Brief, E-Mail und Telefon zu optimieren. Bis zu diesem Zeitpunkt war die Webanwendung ,iRASFF“ (interaktives RASFF) des Schnellwarnsystems für Lebens- und Futtermittel (RASFF-Rapid Alert System for Food and Feed) das einzig praktikable Instrument für einen solchen Austausch. 
Das behördeninterne Informationsnetzwerk RASFF wurde 1979 zum Schutz von Verbraucherinnen und Verbrauchern gegründet, um Informationen zu gesundheitsgefährdenden Lebensmitteln, Lebensmittelkontaktmaterialien oder Futtermitteln schnell und effizient auszutauschen. Die Fälle des Pferdefleischskandals wiesen jedoch keine Gesundheitsgefahr auf und fielen somit aus dem Anwendungsbereich des RASFF heraus. Am 18. November 2015 initiierte deshalb das FFN als spezialisiertes Amtshilfenetzwerk bei Betrugsverdacht die Gründung der Onlineplattform AAC FF (FF: Food Fraud). Das System diente der Übermittlung von Fällen, die mit betrügerischen und irreführenden Praktiken im Lebensmittel- und Futtermittelbereich in Verbindung stehen. Denn eine rechtlich übergreifende und umfassende Definition des Begriffs „Lebensmittelbetrug“ gibt es derzeit nicht. Das FFN hat also vier Kriterien aufgestellt, die erfüllt sein müssen, um von „Lebensmittelbetrug“ zu sprechen:
1. Vorsatz,
2. Verstoß gegen das EU-Lebensmittelrecht,
3. Gewinnerzielung und
4. Täuschung der Verbraucherinnen und Verbraucher.

In 2016 wurde als weitere Onlineplattform das AAC AA (AA: Administrative Assistance) von der Europäischen Kommission ins Leben gerufen, um auch einfache Verstöße gegen das Lebensmittel- und Futtermittelrecht effizienter adressieren zu können. Alle EU-Mitgliedsstaaten, die Europäische Kommission sowie Staaten des Europäischen Wirtschaftsraums sind seit deren Gründung an beiden behördeninternen Netzwerken beteiligt. Über die Europäische Kommission können auch Drittländer über Meldungen informiert werden. Amtshilfe kann in dem Zusammenhang bedeuten, dass Behörden zweier oder mehrerer Mitgliedstaaten Dokumente austauschen, amtliche Kontrollen (ggf. gemeinsam) durchführen oder Vertriebswege nicht verkehrsfähiger Produkte ermitteln. Geht von einem Lebensmittel, Lebensmittelkontaktmaterial oder Futtermittel ein ernstes Gesundheitsrisiko aus, muss jedoch immer das Europäische Schnellwarnsystem (RASFF) aktiviert werden.

\section{Folgemaßnahmen zum Fipronil-Geschehen}

Im Sommer 2017 wurde die Lebensmittelindustrie in Europa durch ein weiteres Ereignis erschüttert-dem Fipronil-Skandal. Die illegale Verwendung des Desinfektionsmittels in Geflügelfarmen führte zu einer Kontamination in Eiern und Geflügelfleisch. Wieder wurde offensichtlich, dass die Kommunikationskanäle und die Nutzung der Warn- und Informationssysteme optimiert werden müssen, um vergleichbare Vorfälle effizienter aufzudecken und die Maßnahmen zu koordinieren. Die Konsequenzen und Lehren zum Geschehen wurden auf einem Treffen der Mitgliedstaaten und der Kommission am 26. September 2017 erörtert. Man einigte sich auf 19 konkrete Maßnahmen für ein konsequenteres Vorgehen der EU gegen Lebensmittelbetrug (European Commission 2018). Unter anderem wurde beschlossen, eine gemeinsame Plattform für das RASFF und das AAC zu entwickeln, um die Kluft zwischen den beiden Systemen zu überbrücken. Außerdem wurde festgelegt, die administrativen Tätigkeiten rund um das RASFF und AAC in einer einzigen Kontaktstelle (Single Contact Point) in den Mitgliedstaaten zusammenzuführen.

\section{Neue Kontrollverordnung (EU) 2017/625 und Zusammenführung der bestehenden digitalen Verfahren zum Informationsaustausch}

Es ist das erklärte Ziel der Europäischen Kommission, die bestehenden Verfahren zum Informationsaustausch im Sinne eines integrierten Informationsmanagementsystems für die Kontrollbehörden entlang der Wertschöpfungskette zu standardisieren und miteinander zu verknüpfen, um Synergieeffekte zu erzeugen. Dieser Ansatz drückt sich in der neuen EUKontrollverordnung (VO (EU) 2017/625 2017) aus. Mit dem Anwendungsbeginn der neuen Verordnung am 14. Dezember 2019 wurde die vorherige Version der Kontrollverordnung (VO (EG) Nr. 882/2004) abgelöst, und auch der sogenannte AAC-Durchführungsbeschluss zur Einrichtung und zum Betrieb des AAC-Systems verlor seine Gültigkeit. An seine Stelle rückte die IMSOC-Verordnung (Durchführungsverordnung (EU) 2019/1715 European Commission 2019). Auf das sogenannte IMSOC—das „Informationsmanagementsystem für amtliche Kontrollen“-—wird in der Kontrollverordnung an zahlreichen Stellen verwiesen: Immer dort, wo es um die Verarbeitung von Daten und den Informationsaustausch geht, soll dies „über das IMSOC“" geschehen (Plischke 2020). Konkrete Details der Umsetzung hat die EU-Kommission in der IMSOC-Verordnung erlassen. Von jetzt an heißt es: Die bestehenden IT-Systeme sollen enger miteinander verknüpft und zu einem umfassenden Informationsmanagementsystem für amtliche Kontrollen entlang der gesamten Lebensmittelproduktionskette zusammengeschlossen werden.

\subsection{Das AAC wird Teil des iRASFF}

Gerade rechtzeitig vor Inkrafttreten der EU-Kontrollverordnung wurde am 22. Oktober 2019 ein wichtiger Schritt getan, einen zentralen Bestandteil der IMSOC-Verordnung umzusetzen: Das Amtshilfeverfahren (AAC AA) wurde in 
Abb. 1 Inkrafttreten der neuen Kontrollverordnung VO (EU) 2017/625 und Integration des AAC-AA und AAC-FF in das iRASFF

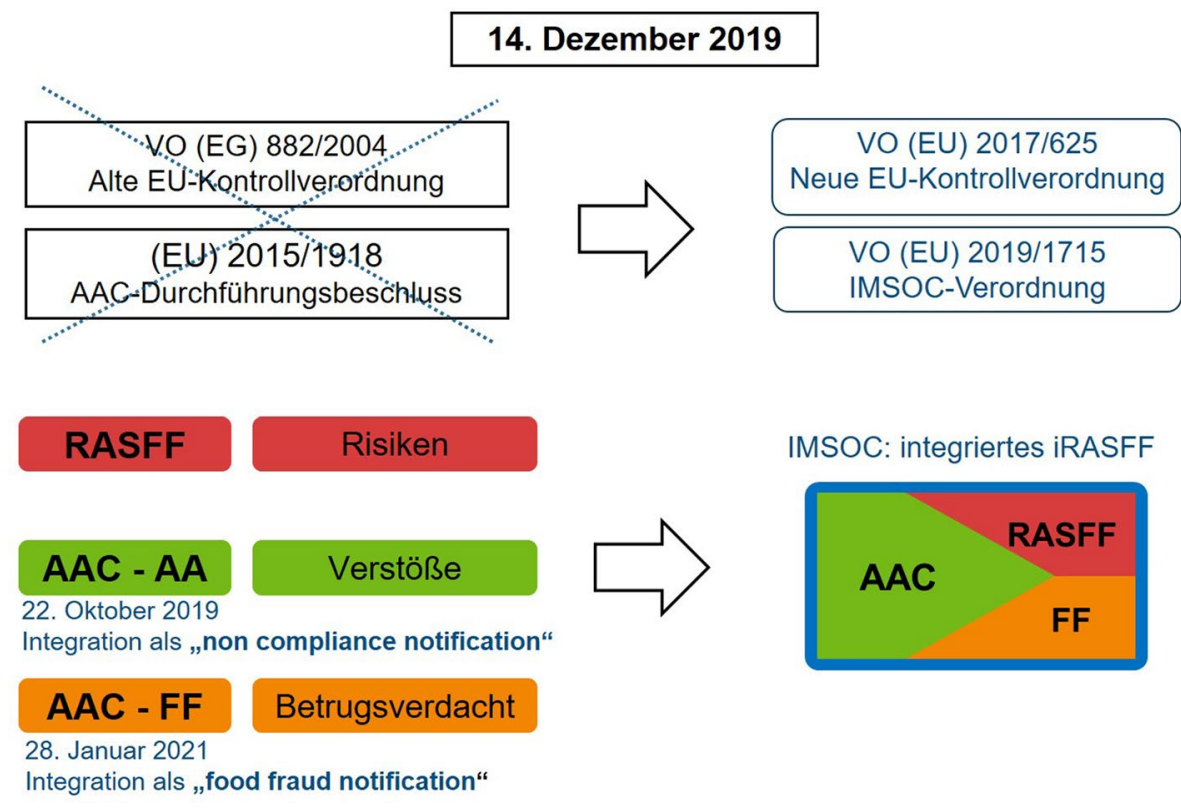

die IT-Plattform iRASFF integriert (s. Abb. 1). Der große Vorteil der Integration liegt in der Verwendung von nur einem System für die Belange der grenzüberschreitenden europäischen Lebensmittel- und Futtermittelkontrolle. So profitiert das AAC-Netzwerk von den bereits etablierten Funktionen des iRASFF, wie der Verknüpfung von Original- und Folgemeldungen, und fügt ihm neue hinzu, wie die Kommunikation über das Konversationsmodul. Der computergestützte Austausch von Informationen zwischen Behörden wird weiter optimiert und die Transparenz der Kontrollergebnisse sowie von amtlichen Entscheidungen erhöht. Durch die enge Verknüpfung der AAC- und RASFFVerfahren wird zudem eine einfachere Eskalation von Fällen ermöglicht, bei denen sich erst im Lauf der Ermittlung z.B. ein Risiko oder ein Betrugsverdacht ergibt (Plischke 2020).

Seit dem erfolgten Start des AAC AA im iRASFF wird die Meldekategorie der Noncompliance bzw. Verstoßmeldung für Sachverhalte genutzt, bei denen grenzübergreifende Verstöße gegen das Lebens- und Futtermittelmittelrecht vorliegen, jedoch keine unmittelbare Gefahr für die Gesundheit von Mensch und Tier besteht. Seit dem 28. Januar 2021 ist das AAC FF Netzwerk mit der Meldekategorie Food Fraud Notification bzw. (Lebensmittel-) Betrugsmeldung ebenfalls in das iRASFF eingebunden, und das ursprüngliche System steht ab dem 30. April 2021 lediglich zur Aufarbeitung bestehender Fälle zur Verfügung.

\subsection{Meldepflicht und ein erweiterter Anwendungsbereich}

Mit Inkrafttreten der neuen Kontrollverordnung und Integration beider AAC-Verfahren besteht die Verpflichtung für die Mitgliedstaaten, für die Verfolgung aller Verstöße grenzüberschreitender Natur sowie Verdachtsfälle von Betrug in der Agrar- und Ernährungswirtschaft das iRASFF zu verwenden (VO (EU) 2017/625). Der Informationsaustausch außerhalb der IT-Plattform, z.B. über E-Mail, verliert immer mehr an Bedeutung. Darüber hinaus erstreckt sich die Meldepflicht nun auf den gesamten Geltungsbereich der Kontrollverordnung (z.B. auch Tiergesundheit, Pflanzengesundheit, Tierschutz). Dementsprechend wird sich der Anwendungsbereich des iRASFF in den nächsten Jahren deutlich ausweiten.

\section{Amtshilfe und Zusammenarbeit in Zahlen}

Erwartungsgemäß startete das AAC System 2016 mit geringen Fallzahlen, wobei diese, gerade im Bereich der Allgemeinen Amtshilfe, über die Jahre sprunghaft anstiegen. Laut der Daten der Europäischen Kommission verdreifachten sich die Meldungen annähernd zwischen den Jahren 2016 und 2019 (vgl. Abb. 2). Im Jahr 2019 wurden etwa 1700 Sachverhalte im Bereich der Allgemeinen Amtshilfe ausgetauscht (Europäische Kommission 2020b). Die meisten dieser Meldungen wurden noch über die ursprüngliche AAC AA-Plattform erstellt. Mit Integration des Netzwerks in die Plattform des iRASFF im Oktober 2019 werden die Fälle zur Allgemeinen Amtshilfe nun als Non-compliance-Meldungen über das iRASFF erfasst. Im Jahr 2020 erzeugten die Netzmitglieder bereits 2248 Verstoßmeldungen, was einer erneuten Steigerung um $33 \%$ gegenüber dem Vorjahr entspricht (Abb. S1 Supplementary Material). Darüber hinaus können seit 2019 sowohl RASFF- als auch AAC-Meldungen mit Konversationen erweitert werden, die zusätzlich zur 
Abb. 2 Gesamtanzahl der in der EU erstellten AAC-Fälle vs. Zahl der Fälle mit Bezug zu Deutschland von 2015-2020

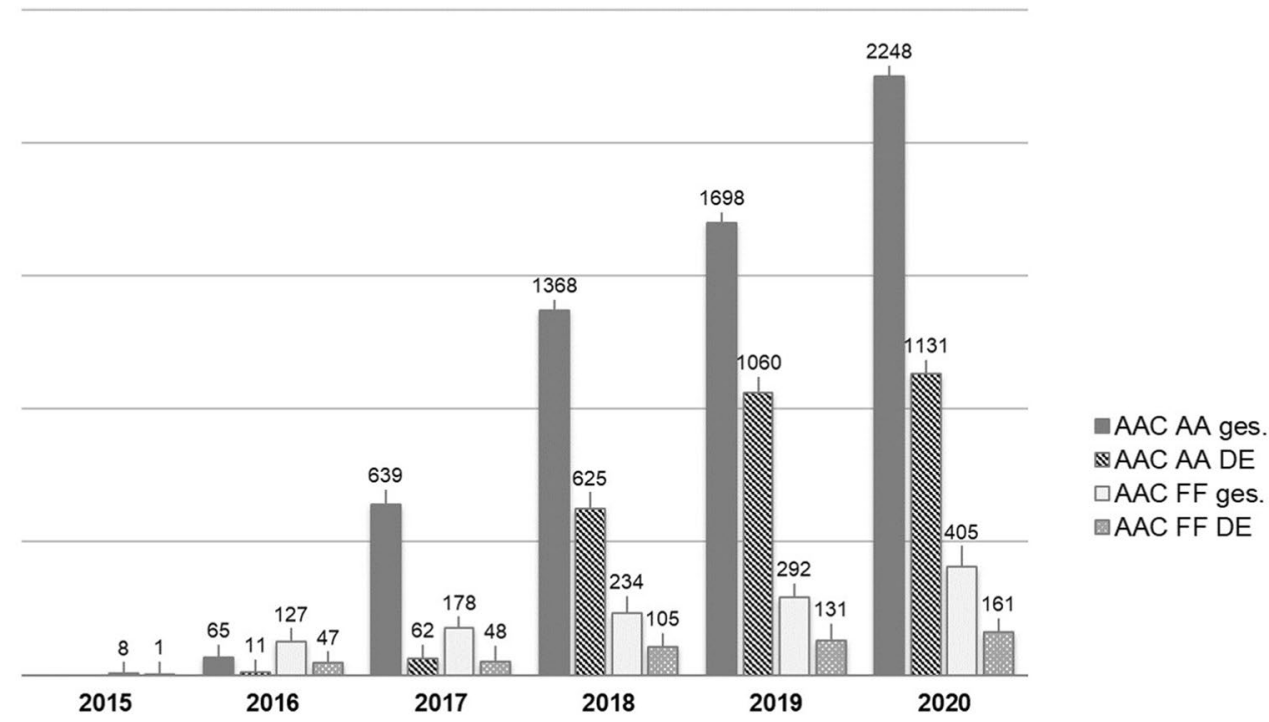

standardisierten Erfassung der Sachverhalte eine direkte Kommunikation zwischen den Netzmitgliedern in Bezug auf die Meldung ermöglichen (European Commission 2020b). Im Jahr 2020 wurden mehr als 10.000 solcher Konversationen im iRASFF geführt (vgl. Abb. S1-Supplemantary Material). Da systembedingt zu jeder AAC Meldung mindestens zwei Konversationen erzeugt werden, fällt der Großteil der Konversationen im AAC Bereich an. Auch die Anzahl der gemeldeten Fälle im Bereich Lebensmittelbetrug nimmt über die Jahre stetig zu (European Commission 2020a). Die Fallzahlen sind jedoch im Vergleich zur Allgemeinen Amtshilfe weitaus geringer und konstanter. Im Jahr 2020 wurden europaweit 405 Fälle eingestellt, was einem Anstieg von fast $40 \%$ zum Vorjahr entspricht.

Seit der Einrichtung der Systeme nutzen v. a. die deutschen Behörden das AAC sehr intensiv (vgl. Abb. 3). Dieser Trend setzte sich auch in 2019 und 2020 in beiden Bereichen fort. Deutschland war mit etwa 800 Fällen in
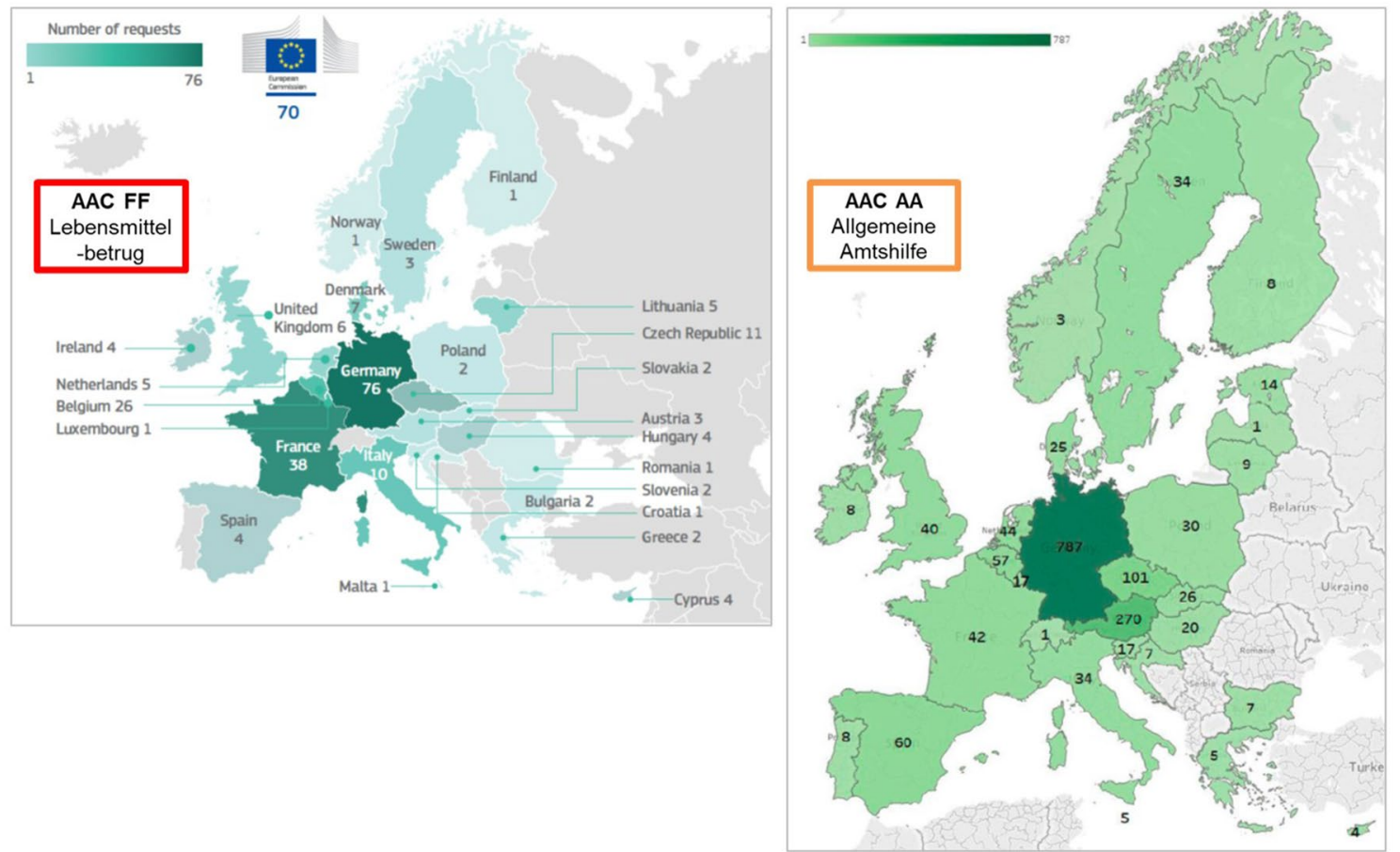

Abb. 3 Gesamtanzahl der erstellten AAC FF und AAC AA Fälle im Jahr 2019 pro Mitgliedstaat, Quelle: Europäische Kommission, Übersetzung \& Aktualisierung BVL (2020) 
beiden Jahren europaweit Spitzenreiter beim Erstellen von AAC AA-Meldungen (2019: 787; 2020: 826). Die recht ungleiche Nutzung der Netzwerke unter den europäischen Mitgliedstaaten ist ein Zeichen für die sich noch entwickelnde Etablierung des verhältnismäßig jungen Netzwerks. Die Meldeverpflichtung durch das Inkrafttreten der neuen Kontrollverordnung im Dezember 2019 wird auch zukünftig zu einem weiteren Anstieg der Fallzahlen führen. Zugleich ist zu beobachten, dass die Netzmitglieder vermehrt mit dem Informationsaustausch auf der Plattform experimentieren und versuchen, herkömmliche E-Mail-Kommunikation zu ersetzen.

\section{Kennzeichnungsmängel als Hauptgrund für Meldungen}

Im Jahr 2020 hatten 1131 AAC AA-Meldungen einen Bezug zu Deutschland, d.h. das dazugehörige Produkt wurde entweder in Deutschland hergestellt oder nach Deutschland geliefert (Abb. S4 Supplementary Material). „Nahrungsergänzungsmittel“ (10\%) und „Getränke“ (9\%) stellten die am häufigsten gemeldeten Produktkategorien mit Bezug zu Deutschland dar. Mit insgesamt 94 Meldungen (8\%) folgt an dritter Stelle die Kategorie „Obst und Gemüse“ (vgl. Abb. 4). Kennzeichnungsmängel bilden mit großem Abstand den Hauptgrund der Beanstandungen. Dies gilt z.B. auch für Nahrungsergänzungsmittel (62\%). Unter diese Kategorie fällt auch die Bewerbung von Produkten mit nicht zugelassenen gesundheitsbezogenen Angaben, sogenannten „Health Claims“ (Abb. S4 Supplementary Material). Fälle bezüglich dieser unzulässigen Auslobungen waren in 2020 häufig Gegenstand der Verstoßmeldungen bei Nahrungsergänzungsmitteln.

In der Produktkategorie „Obst und Gemüse“ betrifft jede zweite Meldung die Beanstandungskategorie ,unzulässige Behandlung und/oder Prozess“. Oftmals handelt es sich hierbei um Überschreitungen des Höchstgehaltes an Pflanzenschutzmittelrückständen ohne Überschreitung der toxikologischen Referenzwerte.
Insgesamt stellen die „Kennzeichnungsmängel“ mit $80 \%$ auch bei der Kategorie der Getränke den Hauptbeanstandungsgrund dar und dominieren damit die Meldungen im AAC.

Auch bei den Lebensmittelbetrugsmeldungen machen die Kennzeichnungsmängel einen großen Anteil bei den Beanstandungsgründen aus. Hier geht es um Angaben, die die Identität der Produkte verschleiern oder eine bessere Qualität vortäuschen. Zwar liegt beim AAC FF in 2020 die Beanstandungskategorie der „Dokumente“ mit 35\% an erster Stelle, jedoch folgt nur knapp dahinter die Kategorie „Falschdeklaration“ mit 32\% der Meldungen (vgl. Abb. S2-Supplemantary Material).

Im AAC FF hatten in 2020 insgesamt 161 Meldungen einen Bezug zu Deutschland. Mit jeweils $18 \%$ stellen die Kategorien „Fisch und Fischereierzeugnisse“ und „Fette und Öle" die am häufigsten gemeldeten Produktkategorien dar (vgl. Abb. S3 - Supplemantary Material). In der Produktkategorie der Fisch und Fischereierzeugnisse wurde am häufigsten ein nicht deklarierter Fremdwasseranteil beanstandet. Durch die fehlende Deklaration entsteht den Herstellern ein wirtschaftlicher Vorteil und der Verbraucher wird bezüglich des Produktgewichtes getäuscht. Bei der Produktkategorie der „Fette und Öle“ wird in fast jeder Meldung die fehlerhafte Benennung und Zusammensetzung beanstandet, zum Beispiel, wenn ein günstiges, mit Chlorophyll eingefärbtes Salatöl als angebliches natives Olivenöl ausgelobt wird.

\section{Fazit}

Das AAC-Netzwerk hat durch die Zusammenführung mit dem Schnellwarnsystem für Lebensmittel und Futtermittel eine positive Entwicklung beschritten. Immer mehr Meldungen werden über die IT-Infrastruktur erstellt. Mit dem iRASFF verfügt das Europäische Netzwerk für Amtshilfe und Zusammenarbeit über ein effizientes und transparenteres digitales Informationsmanagement, mit Vorteilen nicht nur für Behörden, sondern auch für Unternehmen und
Abb. 4 Top-5 der AAC AA Produktkategorien nach Meldungsanzahl in Deutschland 2020

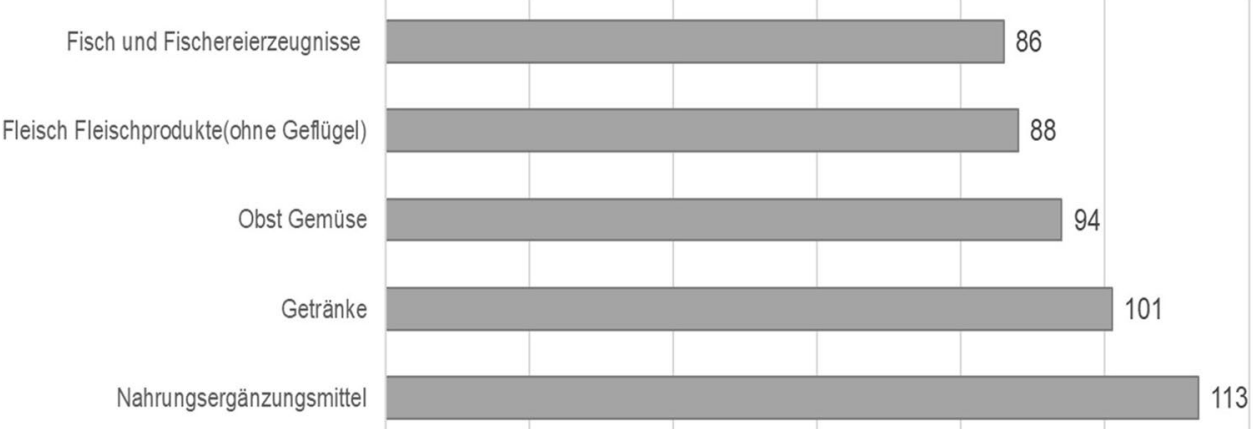


Verbraucherinnen und Verbrauchern. Die digitalen Verfahren stellen einen sicheren Weg des Datenaustausches dar, sie verkürzen die Bearbeitungszeiten, die Verarbeitung von personenbezogenen und Unternehmensdaten wird transparenter und folgt einheitlichen Regeln. Die Verknüpfung der IT-Systeme erhöht die Effektivität und Flexibilität der behördlichen Zusammenarbeit: Vom bilateralen Austausch bei einfachen Verstößen mit grenzüberschreitender Dimension ist es nur ein Mausklick bis zur Alarmierung aller Netzmitglieder, wenn Gefahr im Verzug ist und Risiken schnell begegnet werden muss. Sie schafft darüber hinaus Möglichkeiten, aus vorhandenen Daten neue Erkenntnisse zu gewinnen. Es wird ein zentraler Datenpool über amtliche Kontrollaktivitäten geschaffen, der systematische Verstöße und Risiken offenbaren kann, die möglicherweise von den einzelstaatlichen Kontrollinstanzen nicht erkannt oder nicht effektiv bekämpft werden können. Das iRASFF vernetzt derzeit die Behörden der Lebensmittel- und Futtermittelkontrolle. Der nächste Schritt zur Umsetzung der Ziele der EU-Kontrollverordnung wird die Integration weiterer Kontrollbereiche entlang der Wertschöpfungskette sein. Dies erfordert nicht nur eine grundlegende Überarbeitung der Datenbank, sondern auch ein neues Konzept der Zusammenarbeit von Kontrollbehörden. Jedoch befindet sich die IT-Infrastruktur in einem kontinuierlichen Aufbauprozess und die vorgesehenen Erweiterungen um zusätzliche Systeme laufen mitunter schleppend. Es bleibt abzuwarten, vor welche neuen Herausforderungen, vor allem technischer Natur, alle Beteiligten durch die zukünftigen Systemintegrationen gestellt werden.

Supplementary Information The online version contains supplementary material available at https://doi.org/10.1007/s00003-021-01348-8.

\section{Declarations}

Conflict of interest The authors declare that there is no conflict of interest.

Open Access This article is licensed under a Creative Commons Attribution 4.0 International License, which permits use, sharing, adaptation, distribution and reproduction in any medium or format, as long as you give appropriate credit to the original author(s) and the source, provide a link to the Creative Commons licence, and indicate if changes were made. The images or other third party material in this article are included in the article's Creative Commons licence, unless indicated otherwise in a credit line to the material. If material is not included in the article's Creative Commons licence and your intended use is not permitted by statutory regulation or exceeds the permitted use, you will need to obtain permission directly from the copyright holder. To view a copy of this licence, visit http://creativecommons.org/licenses/by/4.0/.

\section{Literatur}

Durchführungsverordnung (EU) 2019/1715 (2019) der Kommission vom 30. September 2019 mit Vorschriften zur Funktionsweise des Informationsmanagementsystems für amtliche Kontrollen und seiner Systemkomponenten (IMSOC-Verordnung)

European Commission (2018) The EU food fraud network and the system for administrative assistance and food fraud-annual report 2017. https://ec.europa.eu/food/system/files/2020-05/ff_ ffn_annual-report_2017.pdf. Accessed 17 Apr 2021

European Commission (2019) RASFF the rapid alert system for food and feed annual report 2018. https://ec.europa.eu/food/system/ files/2020-05/ff_ffn_annual-report_2018.pdf. Accessed 17 Apr 2021

European Commission (2020a) 2019 Annual Report-the EU Food Fraud Network and the Administrative Assistance and Cooperation System. https://ec.europa.eu/food/system/files_en?file=202005/ff_ffn_annual-report_2019.pdf. Accessed 17 Apr 2021

European Commission (2020b) RASFF the rapid alert system for food and feed annual report 2019. https://op.europa.eu/en/publi cation-detail/-/publication/2c5c7729-0c31-11eb-bc07-01aa7 5ed71a1/language-en/format-PDF/source-174742448. Accessed 17 Apr 2021

Plischke A (2020) IMSOC_-das europäische Digitalpaket. Food Hyg QM 4/2020, pp 14-17

Verordnung (EU) 2017/625 (2017) des Europäischen Parlaments und des Rates vom 15. März 2017 über amtliche Kontrollen und andere amtliche Tätigkeiten zur Gewährleistung der Anwendung des Lebens- und Futtermittelrechts und der Vorschriften über Tiergesundheit und Tierschutz, Pflanzengesundheit und Pflanzenschutzmittel

Publisher's Note Springer Nature remains neutral with regard to jurisdictional claims in published maps and institutional affiliations. 